\title{
User Growth Model of Pinduoduo Based on SIR and Bass Model
}

\author{
Jinling $\mathrm{BAI}^{1, *}$ \\ ${ }^{1}$ Sino-French Institude,Renmin University of China, 158 Renai-Road,Suzhou Industrial District,Jiangsu \\ Province, 215123, China \\ ${ }^{*}$ Corresponding author. Email: baijinling2001@ ruc.edu.cn
}

\begin{abstract}
This study investigates the proper model to predict Pinduoudo's user growth model and the business meaning it contains. Two methods are used in this article: the first one is the Susceptible Infected Recovered model usually used to predict the development of a pandemic, the second one is the Bass model introduced by Frank Bass, which is frequently used to simulate the life cycle of durable products. The results show that the Bass model fits well during the whole developing process of Pinduoduo while SIR is only suitable for the company before its peak. Our finding discovers the Pinduoduo has a two-staged user developing model with a business model combined with traits from both the spread of virus and regular online and offline products, providing a proper prediction model for Pinduoduo's user development as well as a new understanding of its business model for entrepreneurs and investors while filling the gap of this field of research.
\end{abstract}

Keywords: user growth model, Pinduoduo, SIR, Bass

\section{INTRODUCTION}

In the past several years, social e-commerce has been rapidly developing in China and is now playing an important role in the e-commerce market [1]. On one hand, traditional e-commerce platforms' high-growth phase has passed, until the end of 2018, the market size of traditional e-commerce platform reached 8000 billion yuan while the growth rate of the major social platforms such as Taobao, JIngdong was below $20 \%$ and had the tendency to further decline; On the other hand, based on the high traffic of WeChat, social e-commerce was developing rapidly with three major business models: the "group purchase" model, the membership model, the content-based model, among which Pinduoduo developed its unique "group purchase" model and becomes the largest e-commerce platform in the past 6 years [2]. Pinduoduo's most significant business model is "low price+ viral marketing" [3], since its appearance on the market in 2015 [4], Pinduoduo has been using the "cutting price for free" strategy and network marketing to rapidly develop its user amount [5], from 2018 to 2020, Pinduoduo has been maintaining a YOY user growth rate of 53\%, and its market share has also been developing, it is now the second-largest e-commerce platform in China using Gross Merchandise Volumes criteria and the largest e-commerce platform in China using yearly active user (YAU) as criteria. Nowadays, Internet+ digital transformation has become a business trend in most of the fields, in order to gain a healthier developing environment, businesses have to accurately predict future user growth and deploy strategies according to it. As a result, companies should develop their own user growth model which is consistent with the company's special features. But because of the unique business strategy of Pinduoduo, its valuation model and user development model are different from other social media thus leading to a myriad problem of properly calculating and predicting its user development in order to further understand its business model and find potential investment opportunities. A common way to overcome this problem is to use the AARRR model [6], which is wildly used in the mobile market to analyze the user life cycle thus improving the management of the users [7]. But this method requires concise data of the company which is usually kept confidential. An alternative to the problem is to use the SIR model [8], this model was originally used in the research on infectious diseases, such as predicting the peak of a pandemic [9] or discussing a pandemic's development and its economic implications [10], furthermore, SIR model was also used to predict the development of items which have certain similar traits with the infectious diseases such as the spread of computer virus [11] and the spread of an idea in the social 
media [12]. Meanwhile, the Bass model is also suitable to solve this problem, Bass model was first introduced by Frank Bass to predict the growth of durable goods, it was later adapted to analyze the development of fashion clothing [13], pandemics [14] and Internet products [15]. In 2020, Gao Jun and Wang Xinyu [16] used the Bass model to predict the growth of Lingqiantong (an Internet product of WeChat) and had gained great result, proving the Bass model is suitable to be used in Internet product. However, as known, previous research has rarely applied the Bass model to the development of an application or an e-commerce platform, in particular, no previous research has used the Bass model to predict and fit the user development of PInduoduo. Besides, the paper of Cao Jianing in 2019 only used the SIR model to predict the development of Pinduoduo without comparing it to the actual data of Pinduoduo, this may be due to the limitation of time, Pinduoduo was listed on the stock market in 2019 thus they do not have enough data to compare with their prediction model. So it is of interest to know whether the Bass model is still suitable in the field of e-commerce user growth and whether the SIR model's prediction fits the actual data of user development. Bass is the model used to analyze the growth of Internet product or durable product such as fashion clothing while the SIR model is used to simulate the growth of a pandemic. So by researching the usergrowth model, this paper aims to further investigate and confirm the business model of Pinduoduo. That is, whether Pinduoduo only has a virus contagion model or a traditional retailer format, or it shows the characteristics of both the above models. The contributions of this thesis are listed as follows: firstly, it enriches the field of study of Pinduoduo and e-commerce by using the Bass model and SIR model to fit and predict the user growth of Pinduoduo. Secondly, this paper provides a new perspective of Pinduoduo's business model and strategy which can not be simply summarized as virus marketing or traditional Internet product strategy. Thirdly, a model of prediction of user growth for companies like Pinduoduo is provided. With the success of Pinduoduo, many company's decision to develop social e-commerce and draw lessons from PInduoduo's marketing strategy ("group purchase" and social marketing), this paper provides a rather concrete model for the entrepreneurs and investors to forecast the future value of these social e-commerce companies.

\section{METHOD}

\subsection{SIR model}

Referring to [8], the SIR model is used to estimate Pinduoduo's user growth model. SIR model is a pandemic model which can also be used in the spread of information, in which " $\mathrm{S}$ " represents the susceptible, the "I" represents the infected and the "R" represented the recovered. SIR supposes that the total number of people in this area is fixed, if an infected get in contact with a susceptible, there's a chance that the susceptible will be infected, the scale factor is $\beta$; this disease is able to be cured, the scale factor is $\lambda . S(t), I(t), R(t)$ represented the percentage of susceptible, infected and recovered, and the following equations can be got:

$$
\begin{aligned}
& \frac{d S(t)}{d t}=-\beta * I(t) * S(t) \\
& \frac{d I(t)}{d t}=-\beta * I(t) * S(t)-\lambda * \mathrm{I}(\mathrm{t}) \\
& \frac{d R(t)}{d t}=\lambda * \mathrm{I}(\mathrm{t}) \\
& \frac{d S(t)}{d t}+\frac{d I(t)}{d t}+\frac{d R(t)}{d t}=0
\end{aligned}
$$

By solving these equations, $\beta$ and $\lambda$ can be calculated, thus using the scale factors to predict future development.

\subsection{Bass model}

Then, referring to [16], the Bass model is used to estimate Pinduoduo's user growth model. Bass model supposes that there are two channels to spread the product, by social media and by the recommendation of others. In equation 5, p expresses the degree of the effects of social media while q represents the degree of the effects of recommendation. $\mathrm{N}(\mathrm{t})$ is used to indicate the total amount of users and $\mathrm{M}$ to indicate the upper limit of the user amount. From this, the equation can be formulated as follows:

$$
N(t)=M\left[\frac{1-e^{-(p+q) t}}{1+\frac{q}{p} e^{-(p+q) t}}\right]
$$

It shows whether the data is consistent with the development model of Bass and predicts the future development using the scale factor by calculating the $p$ and $\mathrm{q}$ with the existing data. This research uses nonlinear regression to fit the model with the existing data, this method was applied in the article of Qin Yu in 2010 [16].

\section{RESULTS AND DISCUSSION}

\subsection{Data \& Descriptive result}

This research collected the data from Pinduoduo's annual reports so the time period is from 2017 to 2021 . The data is collected each season, the major variables are a yearly active user (YAU) and monthly active user (MAU), the amount is the average amount of the season), the total observation is 16 .

Table 1 Descriptive statistics

\begin{tabular}{lccccc}
\hline Variable & Obs & Mean & Std.Dev. & Min & Max \\
& & & & & \\
\hline yau & 16 & 430.712 & 221.752 & 67.7 & 788.4 \\
mau & 16 & 319.525 & 218.235 & 15 & 720 \\
& & & & & \\
\hline
\end{tabular}




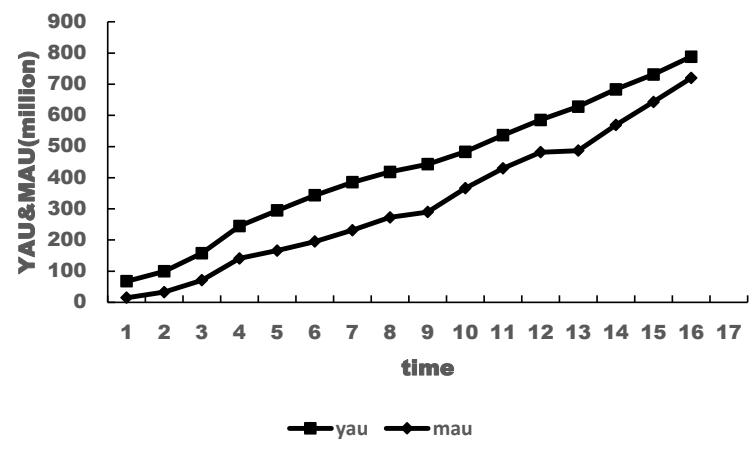

Figure 1 Pinduoduo's YAU\&MAU

On average, the MAU is lower than YAU, YAU and MAU were increasing during the time period but with a decreasing growth rate, this is consistent with the regular development model of e-commerce platforms.

\subsection{SIR model}

Previous studies have indicated that Pinduoduo's traffic is highly related to WeChat's traffic, so the total amount of people in Pindudouo's development model is set as WeChat's YAU in 2019 (1.15 billion), followed by the research of Cao Jianing, $\beta$ is set as $50 \%$ but changed the $\lambda$ to $10 \%$ since in recent years Pinduoduo's MAU/YAU is around $90 \%$, indicating that about $10 \%$ of the users are not fond of Pinduoduo after actively using this app. With the hypothesis set, the predicted development pattern of Pinduoduo has been drawn.

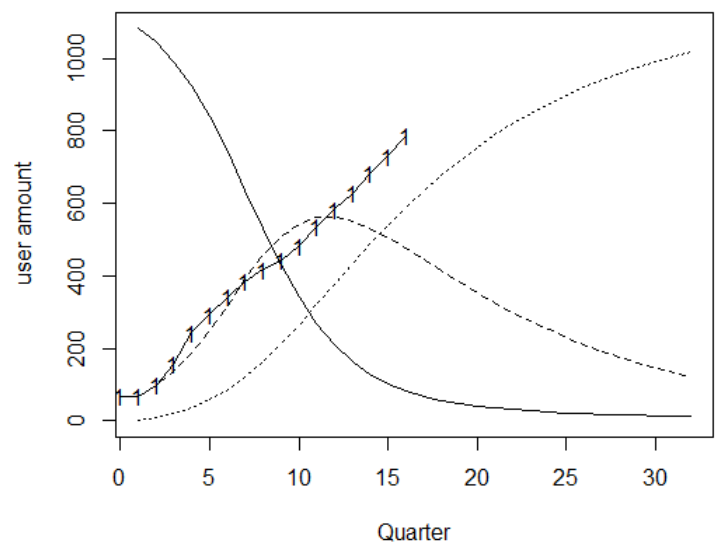

Figure 2 Pinduoduo's user growth prediction

The resrult shows that the SIR model fits the actual model well before the peak of the predicted curve, but Pinduoduo's user amount did not stop developing or start to decrease after the peak, instead, its user amount continued to grow after the predicted peak. This indicates that in the first stage of Pinduoduo's development, by applying the "virus-marketing", Pinduoduo's user development followed the model of SIR. But at the second stage of the development, Pinduoduo applied the "10 billion subsidy" to attract the consumers in first and second-tier cities who the virus marketing could not reach and initiated the "brand supporting plan" to help build inner brands and improve the supply chain management ability to create a better environment for consumers. These actions helped Pinduodou to overcome the tendency to decrease after the peak and kept on growing as an e-commerce platform.

\subsection{Bass model}

Firstly, the data YAU is used to run non-linear analysis to examine if Pinduoduo's user development pattern follows the Bass model, the results are listed in table 2

Table 2 Regression with YAU

\begin{tabular}{lrrrrr}
\hline yau & Coef. & St.Err & t-value & p-value & Sig. \\
\hline$p+q$ & 0.100 & 0.010 & 10.42 & 0.000 & ${ }^{* * *}$ \\
p/q & 1.109 & 0.330 & 3.36 & 0.005 & $* * *$ \\
Mean dependent var & & 430.712 & SD dependent var & & 221.752 \\
R-squared & & 0.998 & Number of obs & 16.000
\end{tabular}

${ }^{\star * \star} p<0.01,{ }^{* *} p<0.05,{ }^{*} p<0.1$

Table 3 Regression with MAU

\begin{tabular}{cccccc}
\hline mau & Coef. & St.Err & t-value & p-value & Sig. \\
\hline
\end{tabular}




\begin{tabular}{lccccc} 
ccons & 0.167 & 0.010 & 16.58 & 0.000 & ${ }^{* * *}$ \\
_cons & 8.053 & 1.262 & 6.38 & 0.000 & ${ }^{* * *}$ \\
& & & & \\
\hline Mean dependent var & 319.525 & SD dependent var & 218.235 \\
R-squared & 0.997 & Number of obs & 16.000 \\
& & & \\
\hline${ }^{* * *} \mathrm{p}<0.01,{ }^{* *} \mathrm{p}<0.05,{ }^{*} \mathrm{p}<0.1$
\end{tabular}

Table 2 represents that the results are statistically significant with an R-squared of 0.998 , indicating that Pinduoduo's user growth model fits in the Bass model with high accuracy. To ensure that our results are not affected by the data, MAU is then used to run NLS analysis, the results are listed in table 3 .

With MAU, the results are also significant with the R-square of 0.997. This indicates that Pinduoduo's growth model is consistent with the Bass model and have a rather high similarity with the Internet products like video games, applications; it also shares a similarity with durable products like fashion clothing, electronic devices and so on.

Based on the results of the SIR model and Bass models, the following results are obtained: firstly, before the peak of the predicted user growth, Pinduoduo's user development model is highly similar to both pandemic development and online product's development. Secondly, after the peak, the SIR model is not consistent with the user development trend of Pinduoduo while the Bass model is still a great fit, both with YAU and MAU data.

\subsection{Discussion}

Before the peak, Pinduoduo used both traditional marketing strategy and virus-marketing. Bass model indicated that the business was using both social media platforms and other media channels as well as relying on the user's recommendation to develop. Two factors indeed both played an important role in Pinduoduo's development: Pinduoduo used other social media platforms such as Weibo and Weixin to promote itself while encouraging its users to recommend the applications to other people. But unlike other ecommerce platforms, Pinduoduo's early development deployed the virus-marketing tactic by which Pinduoduo has made its application a "virus" spreading among potential users. With the traits of pandemic spread and product development, Pinduoduo's early development fitted into both models.

But after the peak, because of the increasing recovered people, the normal pandemic will likely decrease and vanish with time. But as a social platform,
Pinduoduo has the traits of a successful business and social e-commerce platforms, which made it sustainable. By applying the "brand support program", Pinduoduo improved the purchase experience for its users while empowering the online shops and brand in its platform. These actions have been increasing customer loyalty, which, in the SIR model, geared down the appearance of "recover".Pinduoduo also introduced severals luxury brands into the platform to enrich its SKU, using the brands as a natural advertisement to attract customers from higher-tier cities. Besides, Pinduoduo has organized many online and offline events to attract and save customers. After the peak, the "e-commerce platform" side of Pinduoduo was showing, resulting in its data not consistent with the SIR model.

From virus-marketing to e-commerce growth pattern, Pinduoduo kept changing and innovating to accommodate the outside environment and its own development phase. This research predicted that Pinduoduo's development strategy will be more and more similar to normal e-commerce platforms in China like Taobao and JIngdong and using its advantage of high-cost performance to encroach their market share. This is a natural strategy due to its developing phase, Pinduoduo has already passed the fast-developing phase and has entered the steady phase and in this developing phase maintaining a good relationship with the existing users is more important than attracting potential users, by adapting the traditional business model of e-commerce platform, Pinduoduo will be able to improve its priors disadvantages compared with Taobao such as low user consumption. However, virus marketing is a unique advantage of Pinduoduo and it should still deploy this method regarding the promotion of a business segment to attract a large number of users in a relatively short amount of time.

\section{CONCLUSION}

This paper used the SIR and Bass model to fit and predict the future user development of Pinduoduo and gives a relatively better way to forecast the future user growth of Pinduoduo. It has been found that Pinduoduo's development is two-staged, at the beginning stage it presents both the traits of pandemic development and 
online product's/durable product's life cycle. In this stage, Pinduoduo's user growth model fit into both SIR and Bass model. In the second stage, when Pinduoduo has passed the predicted peak, the virus side of Pinduoduo will continue to decrease while the online product side of it will take the dominant role and its development model fits the Bass model well but is not a great fit or SIR model. This finding indicates using a different model in a different stage of the lifecycle of a platform similar to Pinduoduo: in its beginning stage the SIR model can be used to predict its fast growth and use the Bass model to confirm the findings, but when the product is reaching its peak, it is not recommended to use the SIR model and the model should change to Bass model. Besides, this finding helps us realize that Pinduoduo's business model can not be simply concluded as "virus-marketing" or "e-commerce", it is a combination of these two sides and keeps changing depending on the outside and inside situation. The major concern of this study is that the effects of recommendation and advertisement are assumed to remain the same when it may be variable depending on the social circumstance and company's policy as well as time. These factors will be taken into account in the next study. However, from an overall point of view, this study provides new approaches to predict the user development model of Pinduoduo for entrepreneurs and investors while gives a further understanding of Pinduoduo's business model, which was rarely mentioned in this field of study.

\section{REFERENCES}

[1] WANG Ling, Analysis of the development status of China's social e-commerce industry [J].The Internet Economy,2019(Z2):80-89.

[2] HAO Jun-Hui, Huang Zheng "retired", Can Pinduoduo sit firmly on the throne of "the most powerful e-commerce platform" ? [N]. IT times,2021-03-19(002).

[3] WU Sheng-Liang, [J]. Competitiveness Analysis of "PinDuoDuo" E-commerce Platform under the Background of Social E-commerce,2020,36(06):3034.

[4] TENG Yi, Research on the development of network economy $[\mathrm{J}]$. Shanxi Agricultural Economy,2021(04):108-109.

[5] Gang Xu,man Zhang. A Strategy Research of Network Marketing About PinDuoDuo[C]. Institute of Management Science and Industrial Engineering.Proceedings of 2019 9th International Conference on Social Science and Education Research (SSER 2019).Institute of Management Science and Industrial Engineering:Computer
Science and Electronic Technology International Society,2019:1366-1371.

[6] ZHOU Jin-Bo, WEI He-Jun. Research on User Growth Strategy Based on AARRR ModelTaking Buy Together as an Example [J]. Journal of Shanxi Institute of Economic Management,2020,28(01):11-16.

[7] Qianling CHEN and Lan DU, Managing Mobile Market Users Based on the AARRR Model in the Age of Big Data[J]. Management Science and Engineering, 2016, 10(1) : 58-66.

[8] CAO Jia-Ning, WANG Zi-Wen and ZHANG Yu-Xin, User growth model analysis of Pinduoduo platform based on improved SIR model [J]. Modern Business,2019(17):53-57.

[9] TURKYILMAZOGLU Mustafa, Explicit formulae for the peak time of an epidemic from the SIR model[J]. Physica D: Nonlinear Phenomena, 2021, 422

[10] S. G. Babajanyan and Kang Hao Cheong, Agestructured SIR model and resource growth dynamics: a COVID-19 study[J]. Nonlinear Dynamics, 2021, : 1-12.

[11] JIANG Lurong and DAI Yanyun, PAN Hangyi, XU Qiaoyu ,Dong Fang. Research on SIR virus spreading in wireless sensor networks with sleep/listening mechanism [J]. Experimental Technology and Management,2021,38(03):83$87+92$.

[12] QIU Zeguo and YUAN Xiuang, Research on the evolution of network public opinion on short video platform based on improved SIR model [J]. Business \& Economy,2021(02):135-138.

[13] Clothing and Textiles Research; Researchers at Wuxi Institute of Technology Release New Data on Clothing and Textiles Research (A Demand Forecasting Model Based On the Improved Bass Model for Fast Fashion Clothing)[J]. Journal of Technology, 2020,

[14] Kalyanaram Gurumurthy and Avinandan Mukherjee, The Bass Model: a parsimonious and accurate approach to forecasting mortality caused by COVID19[J]. International Journal of Pharmaceutical and Healthcare Marketing, 2020, 14(3) : 349-360.

[15] GAO Jia, WANG Xinyu, Research on the diffusion of Internet financial innovation products - B By the example of WeChat Lingqiantong [J]. Times Finance,2020(29):8-12.

[16] QIN jue, The modification of Bass model and its use in the e-commerce industry, master thesis, Southwest University of science and technology 